\title{
Jefferson Lab phenomenology: selected highlights
}

\author{
W. Melnitchouk ${ }^{\mathrm{a}}$ \\ a Jefferson Lab, 12000 Jefferson Avenue, Newport News, VA 23666, USA
}

An overview of recent experimental highlights from Jefferson Lab is presented. We review the status of baryon spectroscopy, including the search for pentaquarks, as well as measurements of electromagnetic form factors of the nucleon, featuring the proton $G_{E} / G_{M}$ ratio and the determination of the strangeness form factors. In inclusive scattering, we describe recent studies of quark-hadron duality in structure functions in the resonance-scaling transition region, and outline future physics plans at an energy upgraded $12 \mathrm{GeV}$ facility.

\section{INTRODUCTION}

The Thomas Jefferson National Accelerator Facility (Jefferson Lab) is a $6 \mathrm{GeV}$ electron accelerator located in Newport News, Virginia, USA. Since the experimental program began in 1996, Jefferson Lab has developed into the premier electron accelerator facility in the world for precision studies of the structure of the atomic nucleus at the quark level. The Continuous Electron Beam Accelerator Facility (CEBAF) uses superconducting radio-frequency technology to produce an electron beam of unprecedented high luminosity (up to $10^{39} \mathrm{~cm}^{-1} \mathrm{~s}^{-1}$ ) and polarization. The electron beam can be split for use in simultaneous experiments in three experimental halls (Halls A, B and C).

The experimental program at Jefferson Lab touches key problems in nuclear and particle physics, and beyond. These include confinement in QCD, through the study of hadronic spectra and decays; hadronic form factors and quarkgluon distributions (structure functions, generalized parton distributions); strangeness and parity violation in hadrons and nuclei; few-body nuclear physics and the $N N$ force; many-body nuclear physics and the nuclear medium; and finally tests of the Standard Model and beyond. The highprecision data from recent Jefferson Lab experiments are providing stringent tests of theories of hadronic structure, and in some cases forcing reevaluations of commonly accepted wisdom.

Clearly a brief review such as this cannot cover all of these developments; instead we will select a few recent highlights and discuss their impact on our understanding of the structure of hadrons in QCD. We will focus on three main areas: baryon spectroscopy, elastic proton form factors, and inclusive structure functions.

One of the most talked-about developments in hadronic physics in recent years has been the reported discovery of the $\Theta^{+}$pentaquark. We discuss the current status of pentaquark searches at JLab, as well as other results from more conventional excited nucleon spectroscopy. The study of exclusive reactions has provided some fascinating results on electromagnetic form factors of the proton, including the unexpected suppression of the proton electric form factor at large $Q^{2}$. This has led to a re-evaluation of the validity of the one-photon exchange approximation in electronnucleon scattering. Another surprise has been the recent measurements by the HAPPEX and G0 collaborations at JLab of the strange form factors of the nucleon, which when combined with other measurements indicate a large positive value of the nucleon strange magnetic moment.

In inclusive scattering, we review the spectacular observations of quark-hadron duality in inclusive structure functions measured in the nucleon resonance and deep inelastic regions, and what this tells us about the role of multi-quark correlations in the nucleon. Finally, we preview future plans for the $12 \mathrm{GeV}$ energy upgrade of CEBAF, which will include, among other things, the GlueX experiment to look for exotic mesons, and 
precision measurements of quark distributions at large Bjorken- $x$.

\section{BARYON SPECTROSCOPY}

\subsection{Pentaquarks}

The reported observations of pentaquarks in a number of experiments over the past couple of years have produced tremendous excitement in the hadron physics community. As the only baryon with quantum numbers requiring a minimum valence quark content of uudds, the strangeness $S=+1 \Theta^{+}$pentaquark would be the first evidence for an "exotic" baryon in Nature, which cannot be constructed from 3 valence quarks alone.

Following the first observation of the $\Theta^{+}$pentaquark by the LEPS Collaboration [1] at the SPring-8 facility in the inclusive reaction $\gamma n \rightarrow$ $K^{-}\left(K^{+} X\right)$ on a Carbon target, several other experiments reported positive sightings in the mass range around $1.54 \mathrm{GeV}$ and with a width less than a few $\mathrm{MeV}$ (for recent reviews see Refs. [2,3]). This observation was also confirmed by the CLAS Collaboration at Jefferson Lab in photoproduction experiments on deuterium [4] and hydrogen [5].

In response to these reported observations, there has been a plethora of theoretical papers attempting to explain the features of these states. One of the biggest puzzles has been the extraordinarily narrow width, which most experiments have only been able to put upper limits on. Indeed, reanalyses of earlier $K N$ scattering data [6] revealed that a width greater than about $1 \mathrm{MeV}$ would be in conflict with the $K N$ cross sections and phase shifts. It is very difficult to accommodate such a small width within any reasonable theoretical framework or model.

In order to see what QCD predicts for pentaquarks, a number of lattice calculations have been performed to search for pentaquark signals. Unfortunately these have produced conflicting claims about the existence of pentaquarks, with a number of studies claiming positive evidence, while as many others not finding any clear signal, leading to a somewhat confused situation (see Ref. [7] for a recent review). The difficulty lies in identifying precisely what constitutes a resonance on the lattice, and how it can be distinguished from $K N$ scattering states. Although most simulations have focussed on the $J=1 / 2$ channel, an intriguing possibility was raised by Lasscock et al. [8] who found evidence of attraction in the isoscalar $J^{P}=3 / 2^{+}$channel. To draw firm conclusions about whether the observed binding is a finite-volume effect associated with the $N K$ scattering state, a volume-dependent analysis will be needed in this channel.

While the total number of positive sightings accumulated to around a dozen, most of these have been in experiments with relatively low statistics, with uncertainties about estimating the nonresonant backgrounds. Indeed, because the background on top of which the $\Theta^{+}$signal sits is fitted rather than calculated, there have been concerns raised that the reported signal may be due to kinematical reflections [9], or that a different background will produce a dramatically different statistical significance. Along with the 10 or so positive observations, there have been about as many null results, mainly from inclusive highenergy reactions, which have seen no evidence the $\Theta^{+}$state. The resulting experimental situation was thus rather puzzling: if the pentaquarks do exist, it appears that their production mechanism is exotic, along with their quantum numbers.

To bring resolution to this issue, new experiments at JLab have been carried out with around 10 times increased statistics. The g11 experiment in CLAS performed a careful search for a pentaquark signal in the reaction $\gamma p \rightarrow K^{0} K^{+}(n)$ (the neutron was not detected) [10]. In contrast to the earlier results from SAPHIR at ELSA which found a significant $\Theta^{+}$yield in this channel [11], the g11 data found no evidence for a peak in the invariant mass spectrum at the corresponding $\Theta^{+}$mass [12], as Fig. 1 shows.

Furthermore, the g10 experiment in CLAS ran in 2004 to study the reaction $\gamma d \rightarrow p K^{+} K^{-}(n)$. Preliminary results indicate the absence of any noticeable peak in the $p K^{-}$missing mass spectrum. The new data highlight the importance of understanding the nonresonant backgrounds. If one refits the new g10 data with a Gaussian background and uses this to refit the old g2a data, the 


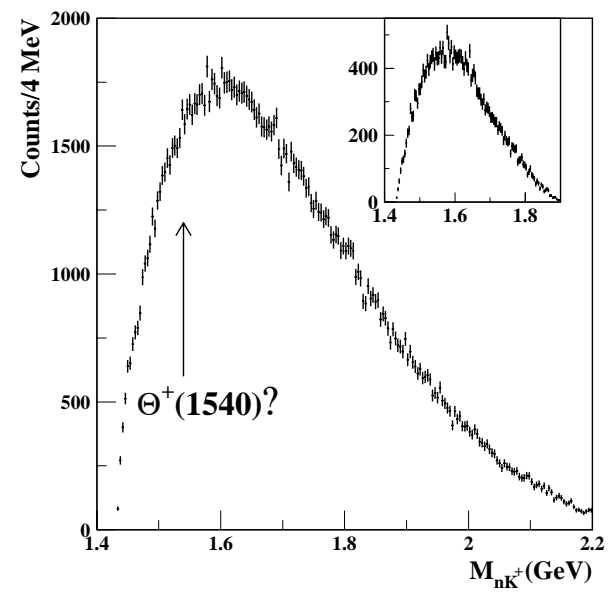

Figure 1. The $n K^{+}$invariant mass distribution, showing no narrow structures [10]. The arrow shows the position where evidence for the $\Theta^{+}$was found by previous experiments. The inset shows the $n K^{+}$mass distribution with specific cuts to reproduce the SAPHIR analysis [10].

statistical significance of the previous peak is reduced from $5.2 \sigma$ to less than $3 \sigma$ [13].

Overall, the evidence in favor of a narrow pentaquark is fading, although it is too early to pronounce its death just yet [12]. Other pentaquark experiments which are planned at JLab (CLAS and in Hall A), SPring-8, and COSY-2 (in $p p$ scattering) should hopefully provide definitive resolution.

\subsection{Excited Baryons}

While considerable attention has been devoted to the pentaquarks searches, other more "mainstream" areas of baryon spectroscopy are also being actively studied at Jefferson Lab. An example is the recent evidence from CLAS for a possible new $P_{13}$ excited nucleon state with mass $\sim 1700 \mathrm{MeV}$ [14], which does not appear to be compatible with the properties of the known $N^{*}$ states in this mass region. The conclusion of these studies is that either the couplings of the known $P_{13}(1720)$ state need to be drastically changed from those given in the Particle Data Book, or a new $P_{13}$ state must be introduced.

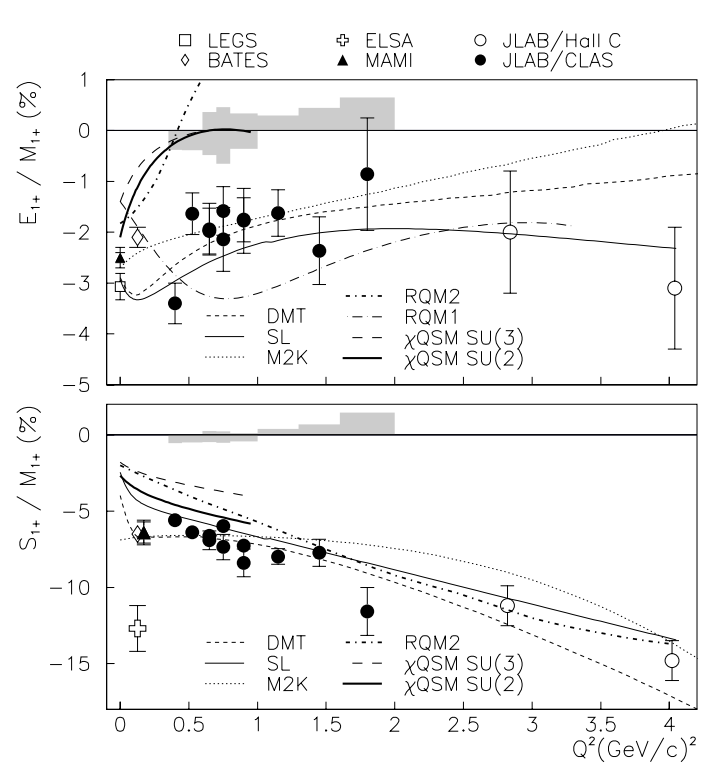

Figure 2. $Q^{2}$ dependence of the ratios of electric $\left(E_{1+}\right)$ and scalar $\left(S_{1+}\right)$ quadrupole amplitudes to the magnetic $\left(M_{1+}\right)$ dipole amplitude, compared with some model calculations [16]. Shaded bands show systematic errors.

Furthermore, an extensive study is underway of $N \rightarrow N^{*}$ transition form factors extracted from meson production data [15]. The simplest of these is the $N \rightarrow \Delta$ transition form factor, which is characterized by the magnetic $\left(M_{1+}\right)$ dipole, and electric $\left(E_{1+}\right)$ and scalar $\left(S_{1+}\right)$ quadrupole amplitudes. In particular, there are rigorous predictions from perturbative QCD (pQCD) for ratios of these amplitude, in particular that in the limit of large $Q^{2}, E_{1+} / M_{1+} \rightarrow 1$, and $S_{1+} / M_{1+} \rightarrow$ constant. These have now been measured to $Q^{2} \sim$ $4 \mathrm{GeV}^{2}$. The results for the $E_{1+} / M_{1+}$ ratio, shown in Fig. 2, remain small and negative $(\sim-2$ to $-3 \%$ ), while the $S_{1+} / M_{1+}$ ratio is also negative and continues to drop with $Q^{2}$, which sug- 
gests that one is still far from the $\mathrm{pQCD}$ region [16].

Data on other resonances continues to be analyzed [15] and is yielding important results. For example, the strong longitudinal response for the $N \rightarrow N^{*}(1440)$ Roper resonance transition suggests that the Roper is not a hybrid qqqg state. Moreover, the results indicate that existing quark model calculations generally fail to simultaneously describe transitions to the $P_{11}(1440)$, $D_{13}(1520)$ and $S_{11}(1535)$ transition amplitudes, so that new directions on the theoretical front are needed.

\section{ELASTIC FORM FACTORS}

Electromagnetic form factors are the most fundamental observables which characterize the composite nature of the nucleon. After several decades of form factor experiments with electron beams, including recent high-precision measurements at Jefferson Lab and elsewhere, considerable information has been accumulated on both the electric and magnetic form factors of the nucleon.

In the one-photon exchange (Born) approximation, the reduced elastic $e N$ cross section can be written in terms of the Sachs electric $\left(G_{E}\right)$ and magnetic $\left(G_{M}\right)$ form factors as

$\sigma_{R}=G_{M}^{2}\left(Q^{2}\right)+\frac{\varepsilon}{\tau} G_{E}^{2}\left(Q^{2}\right)$,

where $Q^{2}$ is (minus) the squared four-momentum transfer, $\varepsilon=\left(1+2(1+\tau) \tan ^{2}(\theta / 2)\right)^{-1}$ is the virtual photon polarization, and $\tau \equiv Q^{2} / 4 M^{2}$.

\subsection{Proton $G_{E} / G_{M}$ Ratio}

The standard method which has been used to determine the electric and magnetic form factors has been the Rosenbluth, or longitudinaltransverse (LT), separation method. Because the form factors in Eq. (1) are functions of $Q^{2}$ only, studying the cross section as a function of the polarization $\varepsilon$ at fixed $Q^{2}$ allows one to extract $G_{M}^{2}$ from the $\varepsilon$-intercept, and the ratio $G_{E} / G_{M}$ from the slope in $\varepsilon$. The results of the Rosenbluth measurements for the proton have generally been consistent with $\mu_{p} G_{E}^{p} / G_{M}^{p} \approx 1$ for $Q^{2}<6 \mathrm{GeV}^{2}$
[17-19], where $\mu_{p}$ is the nucleon magnetic moment, as Fig. 3 illustrates.

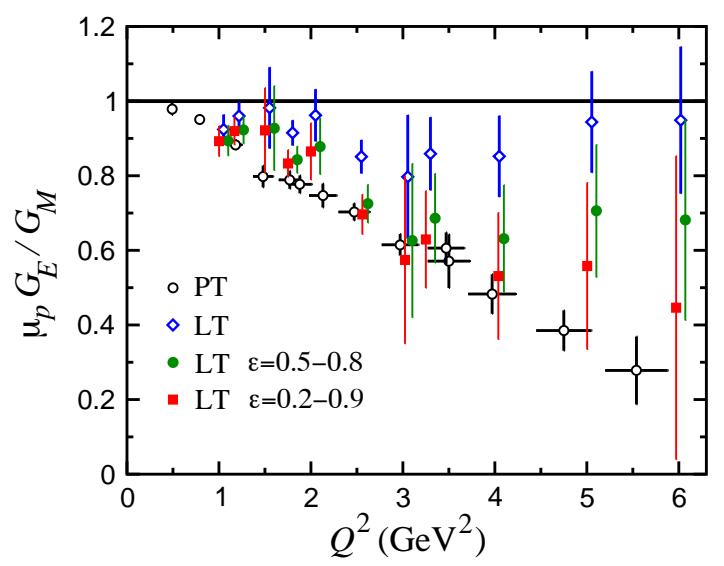

Figure 3. Ratio of the proton form factors $\mu_{p} G_{E} / G_{M}$ measured using LT separation and polarization transfer (PT) [20]. The LT points corrected for $2 \gamma$ exchange are shown assuming a linear slope for two ranges of $\varepsilon$ (offset for clarity) [21].

The alternative method of extracting the ratio $R$, in which a polarized electron beam scatters from an unpolarized target with detection of the polarization of the recoiling proton, determines $R$ from the ratio of transverse to longitudinal recoil polarizations,

$\frac{G_{E}}{G_{M}}=-\frac{P_{t}}{P_{l}} \frac{E_{i}+E_{f}}{2 M} \tan \frac{\theta}{2}$,

where $E_{i}\left(E_{f}\right)$ is the initial (final) electron energy, and $P_{t}\left(P_{l}\right)$ is the polarization of the recoil proton transverse (longitudinal) to the proton momentum in the scattering plane. The polarization transfer (PT) experiments yielded a dramatically different $Q^{2}$ dependence of $R$ than the Rosenbluth [17], as illustrated in Fig. 3. This is in striking contrast to the expectation in $\mathrm{pQCD}$ that at large 
$Q^{2} G_{E} \sim G_{M} / \mu_{p}$, so that these results imply a strong violation of perturbative scaling behavior.

Attempts to reconcile the LT and PT measurements have been made by several authors [2123], who have investigated the possibility that $2 \gamma$-exchange effects, which form part of the radiative corrections, may account for at least part of the observed discrepancy. Although the overall magnitude of the $2 \gamma$-exchange contribution is suppressed by order $\alpha$ relative to the Born term, in practice a change in the $\varepsilon$ slope of $5-6 \%$ would be sufficient to bring the two measurements into agreement [24].

One should note that part of the $2 \gamma$-exchange contributions (the "box" and "crossed box" diagrams) are included in the standard radiative correction analyses [25], using the employs the softphoton approximation (SPA), in which one of the two photons in the internal loop is placed on its mass shell. The SPA makes the contribution factorizable into a product of the Born term and an overall multiplicative factor, effectively neglecting the structure of the nucleon. A partial attempt to go beyond the soft-photon approximation was made in Ref. [26], and a full calculation taking the finite size of the nucleon into account was made by Blunden et al. [21].

While the SPA preserves the linearity of the $\varepsilon$ plot, allowing one to extract $G_{E} / G_{M}$ from the linear slope in $\varepsilon$, the full calculation of the $2 \gamma$ contribution including effects of nucleon structure in principle gives rise to nonlinearity in $\varepsilon$. To estimate the effect of the $2 \gamma$-exchange corrections on the electric to magnetic form factor ratio, Blunden et al. approximate the full $\varepsilon$ dependence by a linear function over a limited range of $\varepsilon$, where the nonlinearity is not large, for each value of $Q^{2}$ at which the ratio is measured. This approximation becomes progressively worse with increasing $Q^{2}$, but is reasonable up to $Q^{2} \sim$ few $\mathrm{GeV}^{2}$. In the actual data analyses one should apply the full $2 \gamma$ correction directly to the data.

The effect of the $2 \gamma$-exchange on the $G_{E} / G_{M}$ ratio is illustrated in Fig. 3 by the filled symbols, corresponding to two different ranges over which the $\varepsilon$ dependence is taken to be linear $(\varepsilon=0.5-$ 0.8 and $\varepsilon=0.2-0.9)$. The $2 \gamma$ corrections are significant, and within errors can resolve most of the discrepancy between the LT and PT results.

The corrections in Fig. 3 correspond to nucleon elastic intermediate states only, and a natural question to ask is what effect higher mass intermediate states may have on the corrected ratio. Contributions from the $\Delta$ resonance have recently been computed by Kondratyuk et al. [27], who found the corrections to have the opposite sign compared with the nucleon, but much smaller in magnitude. Consequently the $\Delta$ contribution has the effect of canceling some of the nucleon correction, including some of the nonlinearity, although the final agreement with data is still very good [27]. Contributions from even higher-lying states, such as the Roper $N^{*}(1440)$ and the odd-parity $N^{*}(1535)$, have the same slope as the $N$ but are very small numerically [28].

The effect of $2 \gamma$-exchange has also been calculated for the PT experiments. The correction to the ratio of the longitudinal polarizations, with and without the $2 \gamma$-exchange, is small (since the correction to the longitudinal cross section is similar to that for the unpolarized cross section), while the correction to the transverse $\mathrm{PT}$ ratio is enhanced at backward angles, and grows with $Q^{2}$. However, since the PT experiments typically have $\varepsilon \approx 0.7-0.8$, the shift in the form factor ratio due to the $2 \gamma$ exchange is small, amounting to $<3 \%$ suppression at the larger $Q^{2}$ values, which is well within the experimental uncertainties.

New experiments at Jefferson Lab will measure the $\varepsilon$ dependence of the cross section to look for deviations from linearity in $\varepsilon$, and also measure the individual polarizations $P_{t}$ and $P_{l}$ in Eq. (2) instead of only the ratio. Finally, a new experiment will simultaneously scatter electrons and positrons elastically from a proton target [29]. Since the interference of the $2 \gamma$-exchange and Born diagrams changes sign for electrons and positrons, whereas the Born term does not, this will unambiguously determine the size of the $2 \gamma$ contribution.

\subsection{Strange Form Factors}

The role of the strange sea of the nucleon has been an ongoing puzzle, ever since the measurements of the spin-dependent nucleon structure functions in the late 1980s suggested that 
strange quarks may carry a large fraction of the proton spin. Complementary investigations of strangeness in the nucleon have focussed on strange quark contributions to nucleon elastic form factors, extracted from parity-violating electron-proton asymmetries which measure the interference between electromagnetic and weak neutral currents.

Recently the G0 collaboration in Hall C at JLab has published the results from their forward scattering run [30], which measured combination $G_{E}^{s}+\eta G_{M}^{s}$, where $\eta=(\tau / \varepsilon) G_{M} / G_{E}$, for a range of $Q^{2}$ between $\approx 0.1$ and $1 \mathrm{GeV}^{2}$. The results, shown in Fig. 4 and compared with the earlier low- $Q^{2}$ HAPPEX results from Hall A, indicate an intriguing $Q^{2}$ dependence.

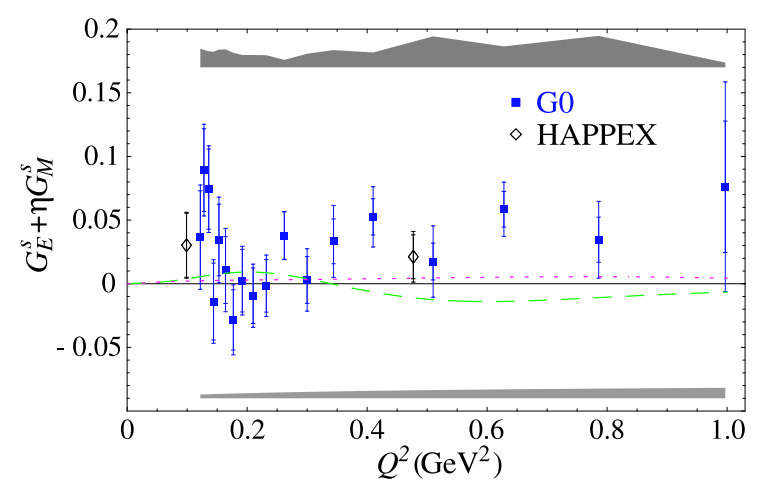

Figure 4. Strange form factor combination $G_{E}^{s}+$ $\eta G_{M}^{s}$ from the G0 experiment [30], together with the earlier HAPPEX data point at low $Q^{2}$. The gray bands indicate systematic uncertainties, and the lines correspond to different nucleon form factor parameterizations used to define the zerostrangeness limit.

When combined with previous data, the G0 results lead to the rather surprising conclusions that the strange magnetic form factor at $Q^{2}=$ $0.1 \mathrm{GeV}^{2}$ is large and positive, $G_{M}^{s}=0.62 \pm$ $0.31 \mu_{N}$, with the strange electric form factor $G_{E}^{s}=-0.013 \pm 0.028$. These results present a challenge to most models of the strangeness content of the nucleon, which generally predict a much smaller magnitude for $G_{M}^{s}$.

In particular, the recent analysis of Leinweber et al. [31], using data on baryon octet magnetic moments, together with lattice QCD simulations of individual quark contributions to magnetic moments and their chiral extrapolations, gives the value $G_{M}^{s}=-0.046 \pm 0.019 \mu_{N}$. This is considerably smaller than the experimental value, which implies a dramatic violation of universality of 70\%. Upcoming experiments at Mainz and JLab, including a new HAPPEX run and the backward angle G0 run, will help to determine whether such a violation is robust, and whether it will require a re-evaluation of our theoretical understanding of the quark structure of the nucleon.

\section{QUARK-HADRON DUALITY}

Elastic form factors provide information about the spatial distributions of quarks in the nucleon. Structure functions, on the other hand, which are measured in inclusive deep inelastic scattering of electrons from nucleons, determine the (lightcone) momentum distributions of quark and gluons (or generically partons) in the nucleon, in terms of the light-cone momentum fraction $x$ of the nucleon carried by the parton. Recent experiments at JLab have provided a number of interesting results in the study of parton distributions and structure functions.

At large $x$ the kinematics of inclusive electron scattering implies that, for fixed $Q^{2}$, one probes deeper into the resonance region at lower final state hadron masses $W, x=Q^{2} /\left(Q^{2}+W^{2}-M^{2}\right)$. The elastic limit, where $W=M$, corresponds to $x=1$. As one goes from the deep inelastic region where the structure functions are smoothly varying functions of $x$, and weakly dependent (approximately scaling) functions of $Q^{2}$, to lower $W$ (higher $x$ ), one encounters the region where oscillations in the structure function data become prominent. The oscillations are associated with the production of nucleon resonances, which appear as peaks and troughs corresponding to the various resonances.

One of the most interesting results to come 
from recent JLab experiments has been the observation that the structure functions measured in the resonance region are on average approximately equivalent to the scaling function which describes the high-energy data. This equality between the resonance region (characterized by hadronic bound states) and the deep inelastic continuum (characterized by scattering from free quarks) is referred to as "quark-hadron duality". First observed by Bloom and Gilman in the late 1960s [32], this duality has been spectacularly confirmed by the new high-precision Jefferson Lab data on the proton $F_{2}^{p}$ structure functions [33]. As illustrated in Fig. 5, the data clearly demonstrate that duality works remarkably well for each of the low-lying resonances to rather low values of $Q^{2}\left(\sim 0.5 \mathrm{GeV}^{2}\right)$, The $F_{2}^{p}$ structure function here is shown as a function of the Nachtmann scaling variable $\xi=2 x /(1+$ $\left.\sqrt{1+4 M^{2} x^{2} / Q^{2}}\right)$, which takes into account kinematical target mass corrections.

Before the advent of QCD, the quark-hadron duality in structure functions was interpreted in the context of finite-energy sum rules, in analogy with the $s$ - and $t$-channel duality observed in hadron-hadron scattering. In QCD, this duality can be reformulated in the language of the operator product expansion, in which moments of structure functions are organized in powers of $1 / Q^{2}$. For the $F_{2}$ structure function, for example, one has for the $n$-th moment

$$
\begin{aligned}
M_{2}^{(n)}\left(Q^{2}\right) & =\int_{0}^{1} d x x^{n-2} F_{2}\left(x, Q^{2}\right) \\
& =\sum_{\tau=2}^{\infty} \frac{A_{\tau}^{(n)}\left(\alpha_{s}\left(Q^{2}\right)\right)}{Q^{\tau-2}},
\end{aligned}
$$

where $A_{\tau}^{(n)}$ are the matrix elements of operators with twist $\leq \tau$ (where twist is defined as the mass dimension minus the spin, $n$, of the operator).

The leading terms in Eq. (4) are associated with free quark scattering, and are responsible for the scaling, while the $1 / Q^{2}$ terms involve interactions between quarks and gluons. The weak $Q^{2}$ dependence of the low moments of the structure function is then interpreted as indicating that the non-leading, $1 / Q^{2}$-suppressed, interaction terms do not play a major role even at low $Q^{2}$.

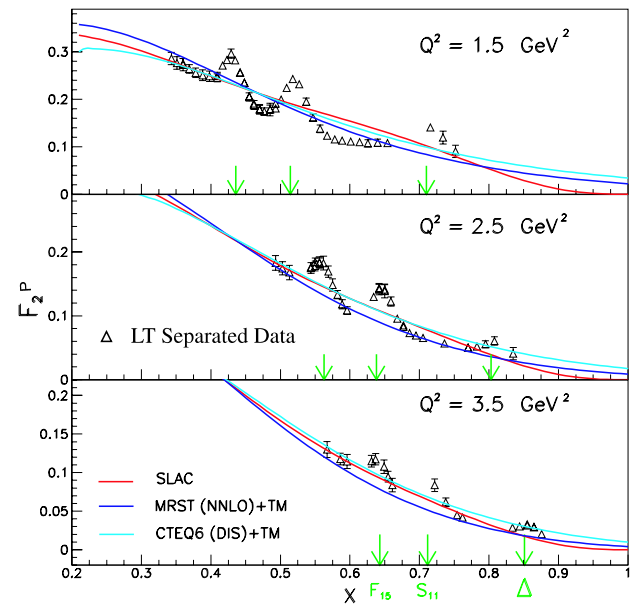

Figure 5. Proton $F_{2}^{p}$ structure function in the resonance region for several values of $Q^{2}$, as indicated. Data from Jefferson Lab Hall C [34] are compared with some recent parameterizations of the deep inelastic data at the same $Q^{2}$ values.

An important consequence of duality is that the strict distinction between the resonance and deep inelastic regions becomes entirely artificial. To illustrate this, consider that at $Q^{2}=1 \mathrm{GeV}^{2}$ around $2 / 3$ of the total cross section comes from the resonance region, $W<2 \mathrm{GeV}$. However, the resonances and the deep inelastic continuum conspire to produce only about a $10 \%$ higher-twist correction to the lowest moment of the scaling $F_{2}$ structure function at the same $Q^{2}$. Even though each resonance is built up from a multitude of twists, when combined the resonances interfere in such a way that they resemble the leading-twist component.

This by itself is quite a remarkable observation. But how can it be made useful in practice? If the degree to which duality holds, or the extent to which duality is violated, is understood, then the resonance data, when properly averaged, can be 
used to extract information on the leading-twist (scaling) parts of structure functions. Furthermore, if the inclusive-exclusive connection via local duality is taken seriously, one can relate structure functions measured in the resonance region to electromagnetic transition form factors.

Conversely, structure function data, and in particular the moments, at intermediate $Q^{2}$ values can be used to extract matrix elements of higher-twist operators. Recent analyses of spindependent structure functions of the proton and neutron have allowed the determination of quantities such as the color-electric and magnetic polarizabilities, which describe the response of the color gluon fields in the nucleon to the spin of the nucleon [35-37]. Although these have been studied to a much lesser extent than the scaling contributions, the higher-twist matrix elements describe long-range, non-perturbative correlations between partons in the nucleon, and as such are as fundamental properties of the nucleon as the spin and momentum carried by quarks.

\section{12 GEV AND BEYOND}

The examples discussed above have only touched the surface of the exciting new physics which has been coming from Jefferson Lab in recent years. Programs to measure few-body form factors at high $Q^{2}$, to map out the quark-hadron transition in exclusive meson-production, as well as new experiments to explore the unchartered territory of generalized parton distributions in deeply virtual Compton scattering, will provide valuable information on the quark structure of hadrons and nuclei in the years to come.

Beyond the next few years, plans to upgrade the energy of the CEBAF accelerator to $12 \mathrm{GeV}$ are under way [38]. The centerpiece of the $12 \mathrm{GeV}$ upgrade will be the construction of a new experimental hall (Hall D), which will enable a systematic search for mesons with exotic quantum numbers, i.e., $J^{P C}$ values which cannot be constructed out of $q \bar{q}$ pairs alone, but which require dynamical glue in the wave function. In addition, the $12 \mathrm{GeV}$ upgrade will permit measurement of deep inelastic structure functions at large values of $x$, beyond anything which has been achievable at laboratories elsewhere, as well as more thorough exploration of the generalized parton distributions.

The future beyond the $12 \mathrm{GeV}$ upgrade may involve a high-luminosity electron-ion collider [39]. This collider - dubbed "ELIC" (Electron-Light Ion Collider) - would require the construction of a new proton ring, into which the electron beam from CEBAF would be injected. One novel element here would be the possibility of operating ELIC as both a collider and in fixed-target mode. Whatever the final outcome of the new extension, it is certain that many exciting experimental results await which will continue to challenge our understanding of nucleon and nuclear substructure.

\section{Acknowledgements}

This work was supported in part by the U.S. Department of Energy contract DE-AC05-84ER40150, under which the Southeastern Universities Research Association operates the Thomas Jefferson National Accelerator Facility.

\section{REFERENCES}

1. T. Nakano et al., Phys. Rev. Lett. 91 (2003) 012002.

2. K. Hicks, Prog. Part. Nucl. Phys. 55 (2005) 647; J. Phys. Conf. Ser. 9 (2005) 183.

3. A. R. Dzierba et al., J. Phys. Conf. Ser. 9 (2005) 192.

4. S. Stepanyan et al., Phys. Rev. Lett. 91 (2003) 252001.

5. V. Kubarovsky et al., Phys. Rev. Lett. 92 (2004) 032001.

6. R. A. Arndt et al., Phys. Rev. C 68 (2003) 042201(R); A. Sibirtsev et al., Phys. Lett. B 599 (2004) 230. W. R. Gibbs, Phys. Rev. C 70 (2004) 045208.

7. F. Csikor et al., hep-lat/0503012.

8. B. G. Lasscock et al., hep-lat/0504015.

9. A. Dzierba et al., Phys. Rev. D 69 (2004) 051901.

10. M. Battaglieri et al., hep-ex/0510061.

11. J. Barth et al., Phys. Lett. B 572 (2003) 127. 
12. V. Burkert, talk presented at $N^{*} 2005$, Oct. 2005, Tallahassee, Florida.

13. S. Stepanyan, talk presented at Pentaquark 2005, Oct. 2005, Jefferson Lab.

14. M. Ripani et al., Phys. Rev. Lett. 91 (2003) 022002 .

15. I. G. Aznauryan et al., hep-ph/0508057; Phys. Rev. C 71 (2005) 015201.

16. K. Joo et al., Phys. Rev. Lett. 88 (2002) 122001; V. V. Frolov et al., Phys. Rev. Lett. 82 (1999) 45.

17. J. Arrington, Phys. Rev. C 68 (2003) 034325.

18. I. A. Qattan et al., Phys. Rev. Lett. 94 (2005) 142301.

19. M. E. Christy et al., Phys. Rev. C 70 (2004) 015206.

20. M. K. Jones et al., Phys. Rev. Lett. 84 (2000) 1398; O. Gayou et al., Phys. Rev. Lett. 88 (2002) 092301.

21. P. G. Blunden, W. Melnitchouk and J. A. Tjon, Phys. Rev. Lett. 91 (2003) 142304; Phys. Rev. C 72 (2005) 034612.

22. P. A. M. Guichon and M. Vanderhaeghen, Phys. Rev. Lett. 91 (2003) 142303.

23. Y. C. Chen et al., Phys. Rev. Lett. 93 (2004) 122301.

24. J. Arrington, Phys. Rev. C 69 (2004) 022201.

25. L. W. Mo and Y. S. Tsai, Rev. Mod. Phys. 41 (1969) 205.

26. L. C. Maximon and J. A. Tjon, Phys. Rev. C 62 (2000) 054320.

27. S. Kondratyuk et al., Phys. Rev. Lett. 95 (2005) 172503.

28. J. Tjon et al., in preparation (2005).

29. JLab experiment E04-116, W. Brooks et al. spokespersons.

30. D. S. Armstrong et al., Phys. Rev. Lett. 95 (2005) 092001.

31. D. B. Leinweber et al., Phys. Rev. Lett. 94 (2005) 212001; A. W. Thomas et al., nuclth/0509082.

32. E. D. Bloom and F. J. Gilman, Phys. Rev. Lett. 16 (1970) 1140.

33. I. Niculescu et al., Phys. Rev. Lett. 85 (2000) 1186.

34. Y. Liang et al., nucl-ex/0410027.

35. Z. E. Meziani et al., Phys. Lett. B 613 (2005) 148.
36. M. Osipenko et al., Phys. Lett. B 609 (2005) 259.

37. X. Ji and W. Melnitchouk, Phys. Rev. D 56 (1997) 1.

38. L. S. Cardman et al., The Science Driving the 12 GeV Upgrade of CEBAF (Jefferson Lab, Newport News, Virginia, 2001).

39. L. Merminga and S. Derbenev, http://casa.jlab.org/research/elic/elic.shtml 